\title{
THE REMAINDER IN APPROXIMATIONS BY MOVING AVERAGES
}

\author{
ARTHUR SARD 1
}

1. Introduction. Many of the processes of interpolation or smoothing are of the following sort. A function $L(s)$, defined for all real $s$, characterizes the process. Given a function $x(s)$, the function

$$
y(t)=\sum_{j=-\infty}^{\infty} x(j) L(t-j)
$$

is constructed, when possible; $y(t)$ is thought of as an approximation of $x(t)$. The remainder in the approximation is

$$
R[x]=x(t)-y(t) .
$$

In the conventional processes of smoothing or interpolation, $L(s)$ is a function which vanishes for all $|s|$ sufficiently large. I. J. Schoenberg ${ }^{2}$ has recently introduced a class of formulas (1), (2) in which $L(s)$ is an analytic function and the series (1) does not consist of a finite number of terms.

Schoenberg gives an elegant criterion for recognizing cases in which the approximating process is exact for polynomials of degree $n-1$; that is, cases in which $R[x]=0$, for all $t$, whenever $x(s)$ is a polynomial of degree $n-1 .^{3}$ In the present paper we obtain an integral representation of such operations $R[x]$ in terms of the $n$th derivative $x^{(n)}(s)$. The representation is precisely of the sort that holds when $R[x]$ is a linear functional on certain spaces of functions $x(s)$ defined on a finite s-interval.

2. The integral representation. We shall consider an operation which is more general than (1), (2). Let $g(s, t)$ be a function which, for each number $t$ in a given set $\mathcal{T}$, is of bounded variation in $s$ on each finite s-interval. Given any function $x(s)$, put 1947.

Presented to the Society, October 25, 1947; received by the editors September 22

1 The author gratefully acknowledges financial support received from the Office of Naval Research, Navy Department.

${ }^{2}$ Contributions to the problem of approximation of equidistant data by analytic functions, Quarterly of Applied Mathematics vol. 4 (1946) pp. 45-99 and 112-141.

${ }^{3}$ Loc. cit. Theorem 2B, p. 64. Schoenberg's criterion is valid whether $L(s)$ is a symmetric function or not.

Throughout the present paper "polynomial of degree $k$ " is to be understood as "polynomial of proper degree $k$ or less." 


$$
y(t)=\int_{-\infty}^{\infty} x(s) d_{s} g(s, t)
$$

and

$$
R[x]=x(t)-y(t), \quad t \in \mathcal{T} .
$$

Unless the contrary is stated, integrals on infinite ranges are to be understood either as Lebesgue-Stieltjes integrals or as improper LebesgueStieltjes integrals, that is, limits of integrals over finite intervals as the intervals become infinite. Either convention may be adopted, providing that it is consistently held. We shall say that $R[x]$ exists if $y(t)$ and $x(t)$ exist and are finite for each $t \in \mathcal{T}$.

The integral (3) reduces to the sum (1) if $g(s, t)$ is, for each $t \in \mathcal{T}$, constant on each interval $j<s<j+1$ and if $g(j+0, t)-g(j-0, t)$ $=L(t-j), j=\cdots,-2,-1,0,1, \cdots$. The name "moving average" is most appropriate to (3) when $d_{s} g(s-m, t)=d_{s} g(s, t+m)$ for all numbers $m$ or for all integers $m$; we do not require that $g$ satisfy this condition.

Assume that $R[x]$ exists and vanishes, for all $t \in \mathcal{T}$, whenever $x(s)$ is a polynomial of degree $n-1(n \geqq 1)$. Put

$$
\begin{array}{rlrl}
p\left(s, s^{\prime}\right) & =\left(s-s^{\prime}\right)^{n-1} /(n-1) ! ; & \\
\psi_{s^{\prime}} & =\psi_{s^{\prime}}(s)= \begin{cases}0 & \text { if } s \leqq s^{\prime}, \\
p\left(s, s^{\prime}\right) & \text { if } s>s^{\prime} .\end{cases}
\end{array}
$$

For each fixed $s^{\prime}, R\left[\psi_{s^{\prime}}\right]$ exists, since $\psi_{s^{\prime}}$ is a truncated polynomial of degree $n-1$. Hence the function $k\left(s^{\prime}, t\right)=R\left[\psi_{s^{\prime}}\right]$ is defined for all $s^{\prime}$ and all $t \in \mathcal{T}$. An alternative formula for $k\left(s^{\prime}, t\right)$ is the following:

$$
k\left(s^{\prime}, t\right)=\left\{\begin{array}{cc}
\int_{-\infty}^{s^{\prime}} p\left(s, s^{\prime}\right) d g(s) & \text { if } s^{\prime}<t, \\
-\int_{s^{\prime}}^{\infty} p\left(s, s^{\prime}\right) d g(s) & \text { if } s^{\prime} \geqq t,
\end{array} \quad t \in \mathcal{T} ;\right.
$$

here 4 and elsewhere $d g(s)$ is to be understood as an abbreviation for $d_{s} g(s, t)$. To establish (5), observe that, if $s^{\prime} \geqq t, \psi_{s^{\prime}}(t)=0$, and

$$
R\left[\psi_{s^{\prime}}\right]=\psi_{s^{\prime}}(t)-\int_{-\infty}^{\infty} \psi_{s^{\prime}}(s) d g(s)=-\int_{s^{\prime}}^{\infty} p\left(s, s^{\prime}\right) d g(s),
$$

by (4). The other part of (5) is derived similarly, with the use of the

${ }^{4}$ Whether the value $s^{\prime}$ is included or excluded in the range of integration of these integrals is immaterial, since $p\left(s^{\prime}, s^{\prime}\right)=0$. 
additional fact that

$$
R\left[p\left(s, s^{\prime}\right)\right]=0=p\left(t, s^{\prime}\right)-\int_{-\infty}^{\infty} p\left(s, s^{\prime}\right) d g(s), \quad t \in \mathcal{T} .
$$

This relation is true because $p\left(s, s^{\prime}\right)$ is a polynomial of degree $n-1$, for each $s^{\prime}$.

Suppose that $x(s)$ is a function whose derivative of order $n-1$ exists and is absolutely continuous on every finite s-interval. Put

$$
\begin{aligned}
I & =\int_{-\infty}^{\infty} d g(s) \int_{0}^{s} p\left(s, s^{\prime}\right) x^{(n)}\left(s^{\prime}\right) d s^{\prime}, \\
R^{*}[x] & =\int_{-\infty}^{\infty} x^{(n)}\left(s^{\prime}\right) k\left(s^{\prime}, t\right) d s^{\prime},
\end{aligned}
$$

Theorem. $A$ necessary and sufficient condition that $R[x]$ and $R^{*}[x]$ exist and be equal is that $I$ exist and that the order of integration in $I$ be invertible, for all $t \in \mathcal{T}$.

Proof. For brevity put

$$
z=p\left(s, s^{\prime}\right) x^{(n)}\left(s^{\prime}\right)
$$

Sufficiency: Since the order of integration in $I$ is invertible.

$$
\begin{aligned}
I=\int_{-\infty}^{\infty} d g(s) \int_{0}^{s} z d s^{\prime}= & -\int_{-\infty}^{0} d s^{\prime} \int_{-\infty}^{s^{\prime}} z d g(s) \\
& +\int_{0}^{\infty} d s^{\prime} \int_{s^{\prime}}^{\infty} z d g(s) .
\end{aligned}
$$

As $x^{(n-1)}(s)$ is absolutely continuous,

$$
x(s)=x(0)+s x^{\prime}(0)+\cdots+\frac{s^{n-1} x^{(n-1)}(0)}{(n-1) !}+\int_{0}^{s} z d s^{\prime} .
$$

Since $R$ vanishes for polynomials of degree $n-1$ and the integral $I$ exists, $R[x]$ exists and

$$
\begin{aligned}
R[x] & =R\left[\int_{0}^{s} z d s^{\prime}\right] \\
& =\int_{0}^{t} p(t, s) x^{(n)}\left(s^{\prime}\right) d s^{\prime}-\int_{-\infty}^{\infty} d g(s) \int_{0}^{s} z d s^{\prime} .
\end{aligned}
$$

Furthermore, 


$$
\int_{0}^{t} p\left(t, s^{\prime}\right) x^{(n)}\left(s^{\prime}\right) d s^{\prime}=\int_{0}^{t} d s^{\prime} \int_{-\infty}^{s^{\prime}} z d g(s)+\int_{0}^{t} d s^{\prime} \int_{s^{\prime}}^{\infty} z d g(s) .
$$

This may be proved as follows. By (6),

$$
p\left(t, s^{\prime}\right)=\int_{-\infty}^{\infty} p\left(s, s^{\prime}\right) d g(s)=\int_{-\infty}^{s^{\prime}} p\left(s, s^{\prime}\right) d g(s)+\int_{s^{\prime}}^{\infty} p\left(s, s^{\prime}\right) d g(s) .
$$

For fixed $t \in \mathcal{T}$, each of the last two integrals is a measurable, essentially bounded function of $s^{\prime}$ for $s^{\prime}$ between 0 and $t$; hence (10) follows.

By (9), (10) and (7),

$$
R[x]=\int_{-\infty}^{t} d s^{\prime} \int_{-\infty}^{s^{\prime}} z d g(s)-\int_{t}^{\infty} d s^{\prime} \int_{8^{\prime}}^{\infty} z d g(s)=R^{*}[x] .
$$

The last equality follows from (5). Thus $R^{*}[x]$ and $R[x]$ exist and are equal.

Necessity: Since $R[x]$ and $R^{*}[x]$ exist and are equal, (11) and (9) hold, and $I$ exists. Furthermore, (11), (9) and (10) imply (7).

This completes the proof of the theorem.

3. Sufficient conditions. Put

$$
M\left(s^{\prime}, t\right)=\left\{\begin{array}{ll}
\int_{-\infty}^{s^{\prime}}\left|p\left(s, s^{\prime}\right)\right||d g(s)| & \text { if } \quad s^{\prime} \leqq 0, \\
\int_{s^{\prime}}^{\infty}\left|p\left(s, s^{\prime}\right)\right||d g(s)| & \text { if } \quad s^{\prime}>0,
\end{array} \quad t \in \mathcal{T} .\right.
$$

If the integral

$$
J=\int_{-\infty}^{\infty}\left|x^{(n)}\left(s^{\prime}\right)\right| M\left(s^{\prime}, t\right) d s^{\prime}
$$

is finite for all $t \in \mathcal{T}$, then $R[x]$ and $R^{*}[x]$ exist and are equal, and $R^{*}[x]$ exists as a Lebesgue-Stieltjes integral.

PROOF. The double integral corresponding to $I$ will exist and (7) will hold, by Fubini's theorem, since the right side of (7) is majorized by $J$. Hence, by the previous theorem, $R[x]$ and $R^{*}[x]$ exist and are equal.

That $R^{*}[x]$ exists as a Lebesgue-Stieltjes integral may be seen as follows. Suppose that $t \geqq 0$. ( $t<0$ is treated similarly.) The integrals

$$
\int_{-\infty}^{0} d s^{\prime} \int_{-\infty}^{s^{\prime}} z d g(s), \quad-\int_{t}^{\infty} d s^{\prime} \int_{s^{\prime}}^{\infty} z d g(s)
$$


are majorized by $J$. Furthermore, by (6),

$$
\int_{0}^{t} d s^{\prime} \int_{-\infty}^{s^{\prime}} z d g(s)=\int_{0}^{t} x^{(n)}\left(s^{\prime}\right) p\left(t, s^{\prime}\right) d s^{\prime}-\int_{0}^{t} d s^{\prime} \int_{s^{\prime}}^{\infty} z d g(s) .
$$

Now the last integral in (13) is majorized by $J$, and the middle integral is on a finite interval. Hence the integrals (12), (13) exist as Lebesgue-Stieltjes integrals. The sum of (13) and the two integrals (12) is precisely $R^{*}[x]$, by (11).

Note that, by (8), the integral (3) will exist as a Lebesgue-Stieltjes integral, in the present case, if it is true that (3) with $x(s)$ a polynomial of degree $n-1$ exists as a Lebesgue-Stieltjes integral.

Anyone of the following conditions is sufficient to imply the finiteness of $J$.

(i) For each $t \in \mathcal{T}, M\left(s^{\prime}, t\right)$ is absolutely integrable and $x^{(n)}\left(s^{\prime}\right)$ is essentially bounded, on $-\infty<s^{\prime}<\infty$.

(ii) For each $t \in \mathcal{T}, M\left(s^{\prime}, t\right)$ is essentially bounded and $x^{(n)}\left(s^{\prime}\right)$ is absolutely integrable, on $-\infty<s^{\prime}<\infty$.

(iii) For each $t \in \mathcal{T}, g(s, t)$ is constant for sufficiently large $s$ and constant for sufficiently small $s$.

In the particular case in which $R[x]$ is of the form (1), (2),

$$
M\left(s^{\prime}, t\right)= \begin{cases}\sum_{-\infty<j \leqq s^{\prime}} p\left(s^{\prime}, j\right)|L(t-j)| & \text { if } \quad s^{\prime} \leqq 0, \\ \sum_{\boldsymbol{s}^{\prime} \leqq j \leqq \infty} p\left(j, s^{\prime}\right)|L(t-j)| & \text { if } \quad \prime^{\prime}>0 .\end{cases}
$$

Queens College 